\title{
Three years of X-ray Light Curve of Swift J164449.3+573451
}

\author{
V. Mangano ${ }^{* a}$, D. N. Burrows ${ }^{a}$, B. Sbarufatti ${ }^{a, b}$, J. K. Cannizzo ${ }^{c, d}$ \\ ${ }^{a}$ The Pennsylvania State University, 525 Davey Lab, University Park, PA 16802, USA \\ ${ }^{b}$ INAF - Osservatorio Astronomico di Brera, Via Bianchi 46, 23807 Merate, Italy \\ ${ }^{c}$ CRESST and Astroparticle Physics Laboratory NASA/GSFC, Greenbelt, MD 20771, USA \\ ${ }^{d}$ University of Maryland, Baltimore County, Baltimore, MD 21250, USA \\ E-mail: vm.himawari@gmail.com
}

Swift J164449.3+573451 was initially detected as a bright and highly variable X-ray transient in March 2011, and soon revealed itself as an object that had no analog in the previous six years of Swift operations. It has been intensively observed over a very broad range of frequencies and interpreted as emission from a jet activated in the tidal disruption event of star in a close-by encounter with a quiescent supermassive black hole in the center of a distant galaxy. Here we present the definitive XRT team light curve for Swift J164449.3+573451 and discuss its implications in the framework of current theoretical models. We show that the light curve decayed roughly as a $\mathrm{t}^{-4 / 3}$ power law for about a year and a half before shut off. The steep turnoff of the jet, dropping an order of magnitude in 24 hours, seems to be consistent with the shutdown of the jet as the accretion disk transitioned from a thick disk to a thin disk. Swift continues to monitor this source in case the jet reactivates.

Swift: 10 Years of Discovery,

2-5 December 2014

La Sapienza University, Rome, Italy

\footnotetext{
* Speaker.
} 


\section{Introduction}

Swift J164449.3+573451 (hereafter Swift J1644+57) triggered the Swift/BAT twice on 2011 March 28th, was automatically repointed at by Swift/XRT (as initially mistaken for a GRB) and, because of its bright and long-lasting X-ray emission, was intensively followed up by Swift/XRT for the first 4 months and then regularly monitored for several years [1]. The prompt and prolonged multi-wavelength follow-up of the new X-ray source contributed substantially to the early recognition of the uniqueness of the event. Swift J1644+57 is considered the first event ever observed from the beginning of tidal disruption (TDE) of a solar-mass star caused by a close passage around a dormant massive black hole (MBH; with estimated mass $\sim 10^{6} \mathrm{M}_{\odot}$ ) in the center of a nearby galaxy (with redshift $\mathrm{z}=0.354$ [2]), in which the emission is dominated by a relativistic expanding jet pointed at us and powered by super-Eddington fall-back accretion of the star debris onto the MBH $[1,2,3,4]$. The XRT light curve in the $0.3-10 \mathrm{keV}$ band shows uninterrupted flaring activity (variability timescales from $\lesssim 100 \mathrm{~s}$ to days, with dynamic range of 1-2 orders of magnitude), a peak after $\sim 1.3$ days (at the flux level of $\sim 9 \times 10^{-9} \mathrm{erg} \mathrm{cm}^{-2} \mathrm{~s}^{-1}$, unabsorbed) and an average decay trend starting from $\sim 7$ days after the trigger [1]. On 2012 August 17th, 508 days after the trigger, the source suddenly dropped below the detection threshold for a single snapshot observation. A 24.7 ks Chandra/ACIS ToO observation performed on 2012 November 26th, $\sim 3$ months after the XRT drop, detected Swift J1644+57 again at the level of $(6 \pm 2) \times 10^{-15} \mathrm{erg} \mathrm{cm}^{-2} \mathrm{~s}^{-1}(0.3-10 \mathrm{keV}$, unabsorbed; [5]). In this paper we present results from a complete reanalysis of Swift/XRT observations of Swift J1644+57 including all data collected up to three years from the original trigger. The complete details of the analysis can be found in [6].

\section{The flux light curve}

The X-ray light curve of Swift J1644+57 in count rate has been extracted in the $0.3-10 \mathrm{keV}$ band as in [1] from all Swift/XRT observations from the trigger to the abrupt drop-off. The remaining 191 monitoring observations performed from 2012 August 17th to 2014 March 28th (total exposure of $284.5 \mathrm{ks}$ ) have been summed together. On this summed image we obtain a significant detection of the source at the count rate level of $1.2_{-0.7}^{+0.9} \times 10^{-4} \mathrm{~s}^{-1}(0.3-10 \mathrm{keV} ; 99 \%$ confidence level). We applied to the final light-curve a refined time-dependent rate-to-flux conversion. A single conversion factor between count rate and flux (ECF) works well for gamma-ray bursts, because there is rarely any significant spectral evolution after the first few ks of the afterglow, but it does not work well for Swift J1644+57, due to the extremely strong spectral variations it shows during the first few hundred days. A more realistic conversion to flux can be obtained via timedependent spectral analysis, i.e. deriving time-dependent ECFs, as we already did in [1]. The conversion applied in [1] was limited to the first 50 days of the Swift J1644+57 outburst, and was based on spectral modeling with an absorbed log-parabola model. Here we complete the set of time-dependent spectra used in [1], reaching a total of 215 spectra, and fit each spectrum with both absorbed power-law and absorbed log-parabola models. The log-parabola model is a curved model, characterized by a photon index and a curvature parameter $\beta$ that has positive values for concave spectra and negative values for convex spectra. When $\beta=0$ the $\log$-parabola reduces to a power-law. Since the two models are suitable for an F-test, we apply it to estimate the probability $\mathrm{P}$ 
of chance improvement of the $\chi^{2}$ and reject all the log-parabola fits with $\mathrm{P}>0.01$. This condition rejects 195 of the log-parabola fits (including all fits with $\beta$ consistent with zero within errors). The log-parabola model significantly improves the fit only for 20 spectra. The final best fit parameters as a function of time are shown in the right panel of Fig. 1. The plot includes the estimated value of the photon index of the post-drop emission obtained by modeling the post-drop spectrum with an absorbed power-law with intrinsic $\mathrm{N}_{\mathrm{H}}$ fixed to $1.9 \times 10^{22} \mathrm{~cm}^{-2}$. The very low statistics did not allow for a fit with the absorbed log-parabola model in this case. Note that the log-parabola best fit models that survive our selection show a strong correlation between $\mathrm{N}_{\mathrm{H}}$, photon index, and $\beta$. This correlation cannot be explained by common properties of the spectra, and we are led to believe that in these 20 special cases the log-parabola fits are merely approximate descriptions that we cannot use for any other purpose than flux estimation.

The time-dependent ECFs for light curve conversion have been calculated as the ratio between the observed flux and the average count rate of each spectrum. The final X-ray light curve of Swift J1644+57 in observed flux $(0.3-10 \mathrm{keV}$ band) is shown in the left panel of Fig. 1. The post-drop point in Fig. 1 is actually consistent with the Chandra detection (converted from [5]).
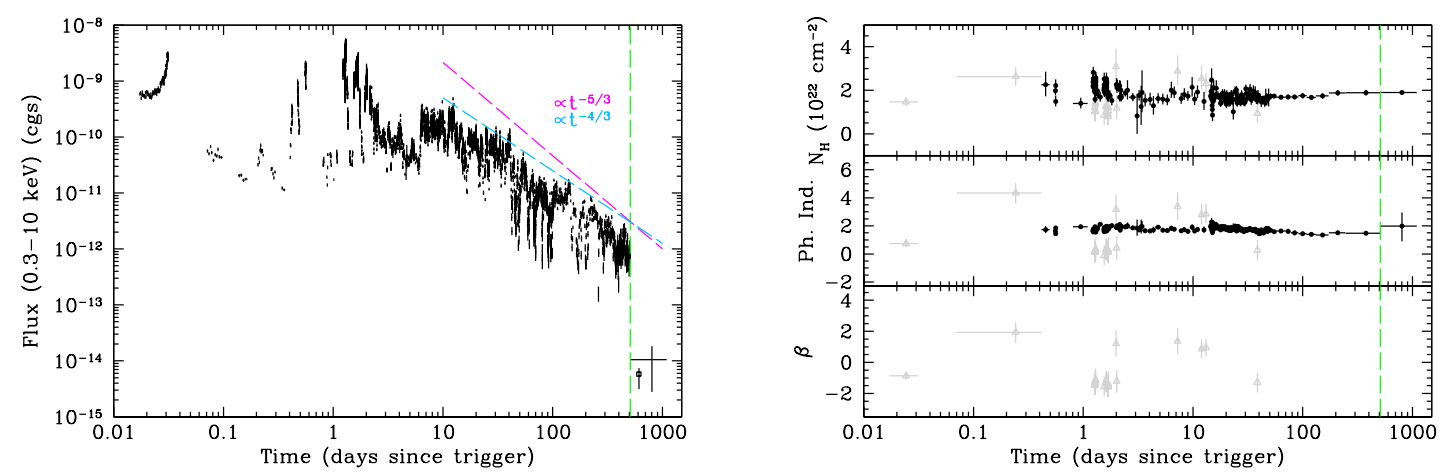

Figure 1: Left panel: X-ray flux light curve of Swift J1644+57 in the $0.3-10 \mathrm{keV}$ band. The vertical green dashed line marks the sudden drop of 2012 August 17th. The open black square marks the Chandra-ACIS $2.8 \sigma$ detection from the $24.7 \mathrm{ks}$ ToO observation performed on 2012 November 26th [5] . For comparison, we show decay rates of $t^{-5 / 3}$ and $t^{-4 / 3}$. Right panel: Temporal evolution of the best-fit parameters of the time-resolved spectra of Swift J1644+57. From top to bottom we plot as functions of time the intrinsic absorption column, the photon index and the curvature of the log-parabola model. Filled circles represent spectra well fit by an absorbed power-law model, while open triangles are used for the 20 spectra for which the absorbed log-parabola model gives a better fit according to F-test as described in the text. The vertical dashed green line marks the drop in the count rate light curve.

\section{The spectral evolution}

A closer look at the temporal evolution of the spectral parameters in the right panel of Fig. 1 shows that for the power-law fits the variations of both the intrinsic $\mathrm{N}_{\mathrm{H}}$ and the photon index approximately track the rate, but with opposite trends: the $\mathrm{N}_{\mathrm{H}}$ tends to be higher at higher rates, while the photon index tends to be lower at higher rates, at least for the early portion of the light curve. These different behaviors are visible in Fig. 2, where the photon indices and the intrinsic 
absorption columns of the time-resolved spectra of Swift J1644+57 well fit by an absorbed powerlaw model are plotted as a function of the $2-10 \mathrm{keV}$ flux. The photon index plot (left panel) shows a structure with a harder-when-brighter correlation progressively shifting leftward in time, i.e. superimposed on a global hardening trend, which becomes dominant at late times. The intrinsic $\mathrm{N}_{\mathrm{H}}$ plot (right panel) has a different structure, with early data (filled circles) showing a moreabsorbed-when-brighter trend, and late data (empty triangles) suggesting a moderately increasing absorption as the flux decays. The intermediate data (empty stars and squares) are more difficult to interpret because of the large dispersion, but seem to follow a trend similar to early data.
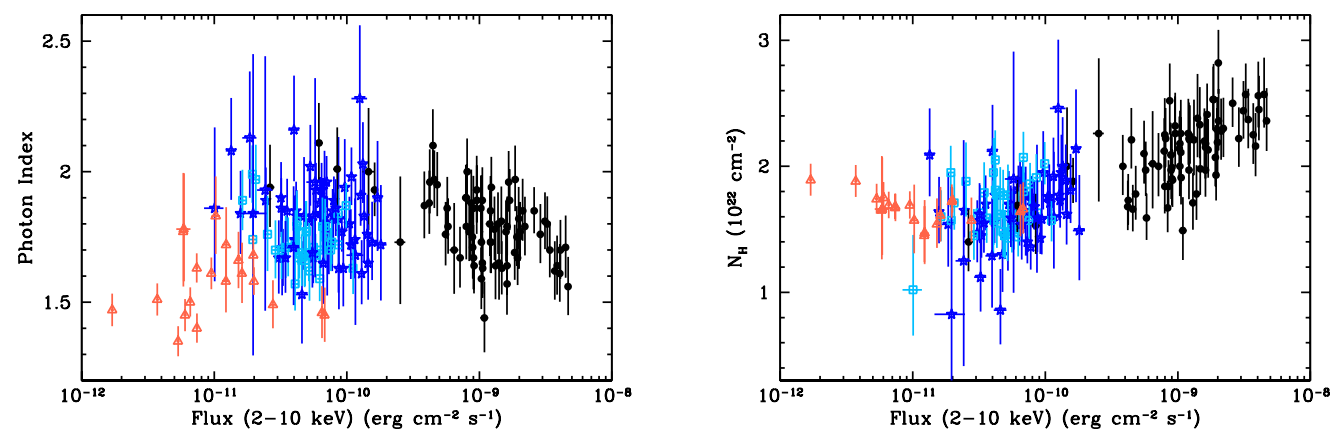

Figure 2: Left panel: Photon indices of the time-resolved spectra of Swift J1644+57 well fit by an absorbed power-law model as a function of the $2-10 \mathrm{keV}$ flux. Right panel: Intrinsic absorption column $\mathrm{N}_{\mathrm{H}}$ as function of the $2-10 \mathrm{keV}$ flux for the same spectra used in the left panel. In both panels filled black circles are data before 2.4 days since the trigger, empty blue stars are data from 2.4 to 23 days since the trigger, empty light blue squares are data from 24 to 39.5 days and empty red triangles are data from 39.5 to 507 days post-trigger.

\section{The light curve decay}

The X-ray light curve of Swift J1644+57 is highly variable but overall, there appears to be a continuous trend underlying the flaring and dipping activity beginning about 6 days after the trigger. We can easily guess by eye a steady power-law like decay after about 10 days and this decay phase seems preceded by a short, flatter plateau phase in the 6.3-10 days period. A single power-law decay with zero reference time shifted by $\Delta \mathrm{t}_{\text {offset }}$ with respect to the trigger may describe the average source emission in the entire time interval as well. The modeling of this global trend is important for the interpretation of the phenomenon. To determine it, we used the following procedure: (1) generate a set of six progressively smoothed light curves by rebinning the flux light curve on a decreasing number of time intervals per decade of days, uniformly spaced in logarithm, according to the sequence $32,16,8,4,3$, and 2 intervals per decade. To each time interval we associate a rebinned point with time and flux coordinates obtained from the arithmetic averages of the logarithms of the times and the fluxes of all the data points therein. (2) fit each smoothed light curve segment within 6 and 508 days since the trigger with a linear model in the logarithm of the time and the logarithm of the flux and derive best fit values for the slope s for every $\Delta \mathrm{t}_{\text {offset }}$ in the $0-45$ days range. (3) calculate the mean and sample variance of the six estimated values of $\mathrm{s}$ 


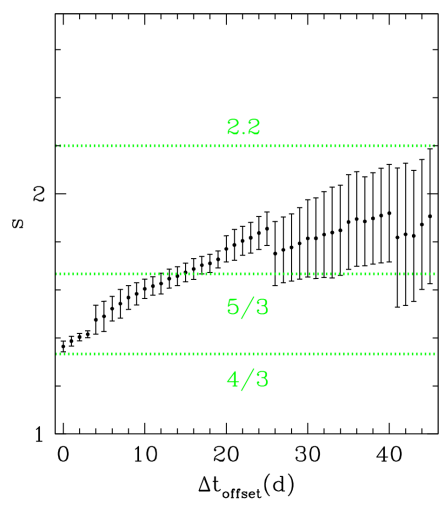

Figure 3: Average power-law decay slope of the flux light curve of Swift J1644+57 for $\mathrm{t}>6$ days posttrigger as a function of the offset of the tidal disruption event onset from the BAT trigger. The horizontal lines at 4/3, 5/3, and 2.2 represent expected values of the slope for different physical models of the disruption event.

for each $\Delta \mathrm{t}_{\text {offset }}$ and use the result as measure of the average slope $\mathrm{s}$ of the flux light curve and its error. The plot of the average slope s versus $\Delta \mathrm{t}_{\text {offset }}$ is shown in Fig. 3. From this plot we see that solutions with $\Delta \mathrm{t}_{\mathrm{offset}}$ of the order of a few days can give an almost equivalent description of the light curve decay rate after about 6 days since the BAT trigger. We interpret the growth of the error on s for larger values of $\Delta \mathrm{t}_{\text {offset }}$ as an indication of lower probability of the $\Delta \mathrm{t}_{\text {offset }}$ time from the BAT trigger being so large. For $\Delta \mathrm{t}_{\text {offset }}=0$ we obtain a final decay slope of $1.36 \pm 0.02$.

According to classical TDE models, the central MBH accretes mass from the disrupted star debris at a rate $\propto \mathrm{t}^{-5 / 3}$, and its luminosity is expected to follow the same temporal decay law. The average slope value we obtain is not consistent with $5 / 3$, but it is rather consistent at the $2 \sigma$ level with 4/3. This slope may also be consistent with the TDE phenomenon when more realistic physics of accretion disks is properly taken into account [7].

\section{Discussion}

We have produced and studied the most accurate and complete X-ray light curve of Swift J1644+57 from three years of Swift/XRT data from its discovery. The X-ray emission after the abrupt drop-off detected by Chandra/ACIS is detected by Swift/XRT as well. The spectral variability of Swift J1644+57 in X-rays across its entire evolution can be described by an irregular harderwhen-brighter behavior tracking flares and dips, with a long term hardening trend associated with the final decay trend. The harder-when-brighter behavior may arise naturally from the interplay between the synchrotron spectral component and Comptonized radiation from the accretion disk.

By fitting the light curve decay slope for 6 days $<\mathrm{t}<508$ days for six logarithmic binnings in $\log \Delta \mathrm{t}$ we determine a post-fluctuation decay slope $\mathrm{s}=1.36 \pm 0.02$. This is statistically distinguishable from the $s=5 / 3$ value for Swift J1644 +57 commonly cited in the literature, but may not be inconsistent with the TDE phenomenon.

In reference [8] the authors suggest that after the fallback time for the most tightly bound debris to return to the $\mathrm{MBH}$ and to begin to accrete $\left(\mathrm{t}_{\mathrm{fb}}\right)$ there may elapse an additional $\Delta \mathrm{t}_{\text {flux }}$ for magnetic flux accumulation in the inner disk to build up to the point that the jet becomes active and we start observing radiation, i.e. $\Delta \mathrm{t}_{\mathrm{offset}}=\mathrm{t}_{\mathrm{fb}}+\Delta \mathrm{t}_{\mathrm{flux}}$. Following [9], we can directly measure $\mathrm{t}_{\mathrm{fb}}+\Delta \mathrm{t}_{\text {flux }}$ as the ratio between fluence and peak flux obtaining $\mathrm{t}_{\mathrm{fb}}<1$ day. The combination of our (i) small inferred $\mathrm{t}_{\mathrm{fb}}$ and (ii) $\mathrm{s}=4 / 3$ support the viewpoint of a rapid transition from stellar fallback to disk accretion, where the disk is highly advective [7,9]. A value $\mathrm{t}_{\mathrm{fb}}<1$ day challenges 
current theory, which favors $\mathrm{t}_{\mathrm{fb}} \sim 20-30$ days, but does not consider strong general relativistic effects in the Kerr metric for large $\mathrm{R}_{\mathrm{T}} / \mathrm{R}_{\mathrm{P}}$ (i.e. disruption radius over periastron radius) encounters; modifications in the binding energy spread for the tidal debris from the standard results for $R_{T} / R_{P}$ $\sim 1$ encounters are treated via linear perturbations to a Newtonian gravitational potential [10], [11, - see their Sect. 6].

\section{Acknowledgments}

This work was supported by NASA grant NNX10AK40G. This work made use of data supplied by the UK Swift Science Data Centre at the University of Leicester. We acknowledge the use of public data from the Swift data archive.

\section{References}

[1] Burrows, D. N. et al., Relativistic jet activity from the tidal disruption of a star by a massive black hole, Natur 476 (2011) 421.

[2] Levan, A. J. et al., An Extremely Luminous Panchromatic Outburst from the Nucleus of a Distant Galaxy, Science 333 (2011) 199.

[3] Bloom, J. S. et al., A Possible Relativistic Jetted Outburst from a Massive Black Hole Fed by a Tidally Disrupted Star, Science 333 (2011) 203.

[4] Zauderer, B. A. et al., Birth of a relativistic outflow in the unusual $\gamma$-ray transient Swift J164449.3+573451, Natur 476 (2011) 425.

[5] Zauderer, B. A. et al., Radio Monitoring of the Tidal Disruption Event Swift J164449.3+573451. II. The Relativistic Jet Shuts Off and a Transition to Forward Shock XRay/Radio Emission, ApJ 767 (2013) 152.

[6] Mangano, V., Burrows, D. N., Sbarufatti, B., Cannizzo, J. K. The definitive X-ray Light Curve of Swift J164449.3+573451, ApJ submitted (2015).

[7] Cannizzo, J. K., Troja, E., Lodato, G., GRB 110328A/Swift J164449.3+573451: The Tidal Obliteration of a Deeply Plunging Star? ApJ 742 (2011) 32.

[8] Tchekhovskoy, A., Metzger, B. D., Giannios, D., Kelley, L.Z. Swift J1644+57 gone MAD: the case for dynamically important magnetic flux threading the black hole in a jetted tidal disruption event, MNRAS 437 (2014) 2744.

[9] Gao, W.-H. SWIFT J164449.3+573451: A Plunging Event with a Poynting-flux-dominated Outflow, ApJ 761 (2012) 113.

[10] Guillochon, J., Ramirez-Ruiz, E. Hydrodynamical Simulations to Determine the Feeding Rate of Black Holes by the Tidal Disruption of Stars: The Importance of the Impact Parameter and Stellar Structure, ApJ 767 (2013) 25.

[11] Stone, N., Sari, R., Loeb, A. Consequences of strong compression in tidal disruption events, MNRAS 435 (2013) 1809. 